\title{
Auricular complications following temporal bone resection for temporal bone malignancies: a clinical consideration
}

\author{
Noritaka Komune $^{1}$, Satoshi Matsuo ${ }^{2}$, Ryo Shimamoto ${ }^{1}$, Kou Ikemura ${ }^{1}$, Joe Iwanaga ${ }^{3}$, Emi \\ Nishimura $^{1}$, Mayu Miyazaki ${ }^{1}$, Chikafumi Oryoji ${ }^{1}$, Shogo Masuda ${ }^{1}$, Takahiro Hongo ${ }^{1}$, \\ Kensuke Koike ${ }^{1}$, Kuniaki Sato ${ }^{1}$, Ryutaro Uchi ${ }^{1}$, Masaru Miyazaki ${ }^{4}$, Nana Akagi \\ Tsuchihashi $^{1}$, Teppei Noda ${ }^{1}$, Ryunosuke Kogo ${ }^{1}$, Sei Yoshida ${ }^{1}$, Hideki Kadota ${ }^{1}$, and Takashi \\ Nakagawa $^{5}$ \\ ${ }^{1}$ Kyushu University Hospital \\ ${ }^{2}$ Hamanomachi Hospital \\ ${ }^{3}$ Tulane University School of Medicine \\ ${ }^{4}$ Fukuoka University Hospital \\ ${ }^{5}$ Kyushu University
}

December 13, 2020

\begin{abstract}
Abstract: Objective: Sacrificing the auricle has severe postoperative impacts on patients with temporal bone malignancy who have undergone en bloc temporal bone resection. The auricular complications following en bloc temporal bone resection remain unclear. Therefore, this study aimed to examine auricular complications following en bloc temporal bone resection and reveal the associated risk factors. Study Design: Case series with chart review. Setting: Single tertiary care medical center Participants: Our study includes all cases who underwent en bloc temporal bone resection with preserved auricle for temporal bone malignancy from January 1993 to July 2020. The resulting dataset contained 64 patients during the review period. Main outcome measures: We examined the relationship of auricular complications with surgical procedures and patient profiles. Results: Three of 64 cases $(4.7 \%)$ showed postoperative auricular complication. The cause in all three cases was venous congestion. Postoperative bloodletting to relieve the congestion effectively salvaged the auricle in two of these cases. Insufficient bloodletting to relieve the auricular congestion in the remaining case led to auricular necrosis. Pre-/infra-auricular lymph node dissection is correlated with auricular complications with statistical significance. Conclusions: Temporal bone resection causes auricular congestion due to vascular insufficiency, particularly insufficient venous outflow. An immediate and continuous application of bloodletting can improve venous drainage after temporal bone resection to prevent auricle necrosis.
\end{abstract}

\begin{abstract}
:
Objective: Sacrificing the auricle has severe postoperative impacts on patients with temporal bone malignancy who have undergone en bloc temporal bone resection. The auricular complications following en bloc temporal bone resection remain unclear. Therefore, this study aimed to examine auricular complications following en bloc temporal bone resection and reveal the associated risk factors.

Study Design: Case series with chart review.

Setting: Single tertiary care medical center

Participants: Our study includes all cases who underwent en bloc temporal bone resection with preserved auricle for temporal bone malignancy from January 1993 to July 2020. The resulting dataset contained 64 patients during the review period.
\end{abstract}


Main outcome measures: We examined the relationship of auricular complications with surgical procedures and patient profiles.

Results: Three of 64 cases (4.7\%) showed postoperative auricular complication. The cause in all three cases was venous congestion. Postoperative bloodletting to relieve the congestion effectively salvaged the auricle in two of these cases. Insufficient bloodletting to relieve the auricular congestion in the remaining case led to auricular necrosis. Pre-/infra-auricular lymph node dissection is correlated with auricular complications with statistical significance.

Conclusions: Temporal bone resection causes auricular congestion due to vascular insufficiency, particularly insufficient venous outflow. An immediate and continuous application of bloodletting can improve venous drainage after temporal bone resection to prevent auricle necrosis.

\section{Key Point:}

- The preservation of the auricle in temporal bone resection is as important as the prevention of other complications, such as facial nerve weakness/paralysis.

- Temporal bone resection can cause auricular congestion due to insufficient venous outflow in head and neck region.

- $4.7 \%$ of temporal bone resection cases showed postoperative auricular complication.

- Pre-/infra-auricular lymph node dissection can be correlated with auricular complications

- An immediate and continuous application of bloodletting can replace insufficient venous drainage after temporal bone resection and prevent auricle necrosis.

\section{Introduction:}

The frequency of temporal bone malignancies is extremely low.(1) The two most common types are squamous cell carcinoma and adenoid cystic carcinoma. A lack of research has hindered the development of suitable treatment guidelines. According to the literature, margin-negative and en bloc resection are gold standards for treatment. Unless the tumour extends directly to the auricle, preserving the auricular is important for en bloc temporal bone resection to maintain quality of life among patients. Unfortunately, few reports investigate auricular complications during en bloc temporal bone resection. (2)

En bloc resection of the temporal bone involves two common procedures: lateral temporal bone resection (LTBR) and subtotal temporal bone resection (STBR). Surgeons assess T and N stages to combine procedures, including parotidectomy, neck dissection, and mandibular condylectomy. Extensive temporal bone resection, i.e. STBR and LTBR combined with parotidectomy, sacrifices blood flow of the auricle. However, auricular complications following en bloc temporal bone resection remain unclear.

Auricular complications lead to additional surgical procedures, such as debridement and delayed adjuvant therapy. Further, auriculectomy has significant psychological and cosmetic impacts on the individual, resulting in poorer quality of life. Therefore, preserving the auricle is important for en bloc temporal bone resection.

In this study, we examined the vascular anatomy of the auricle in cadavers and retrospectively investigated auricular complications in en bloc temporal bone resection preserving the auricle to reveal the risks with vascular insufficiency in the auricle.

\section{Participants and Methods}

Ethic statement

Our study was approved by both ethics review committees of Kyushu University Hospital (permit no. 29-43).

Patient selection and profile

We retrospectively reviewed patient records, as shown in the patient selection flow chart in Figure 1. Applicable patients were treated at the Department of Otorhinolaryngology Head and Neck Surgery, Kyushu 
University Hospital, from January 1993 to July 2020. The initial dataset of patients with temporal bone malignancies comprised 181 cases, of which a final dataset of 64 cases was selected based on patients treated with en bloc temporal bone resection preserving the auricle. To estimate patient physical functioning, we used the Eastern Cooperative Oncology Group (ECOG) performance status scale. All patients were capable of self-care and their performance status was defined as [?] 2.

Data collection and statistical analysis

From the final data set, we gathered information related to auricular complications, including sex, age, affected side, clinical stage, presence of pre-/infra-auricular lymph node dissection, history of radiotherapy, surgical procedure, type of skin incision, additional surgical procedures, reconstruction method, and auricular complications. We used a Fisher exact two-sided test to examine the association between auricular complications and categorical variables, namely neck dissection, parotid gland resection, pre-/infra-auricular lymph node metastasis, temporomandibular joint manipulation, and history of radiation therapy at the locoregional site. We used JMP 6.1 software (SAS Institute, Cary, NC, USA) for statistical analysis and selected $\mathrm{P}<0.05$ to indicate statistical significance.

Cadaveric dissection

Three formalin-perfused adult cadaveric heads were dissected to reveal the vascular structures related to the auricle. The arteries and veins were injected with red or blue silicone rubber (Dow Corning, Midland, MI, USA), Thinner 200 (Dow Corning), and RTV catalyst (Dow Corning), then dissected under 3-40 $\times$ magnification.

En bloc surgical procedure for temporal bone malignancies

Two surgical procedures are used globally: 1) lateral temporal bone resection, which involves en bloc removal of the external auditory canal (EAC) along with the tympanic membrane; and 2) subtotal temporal bone resection, which involves en bloc removal of the temporal bone, including (or transecting) the otic capsule. Depending on local progression, these are combined with additional procedures, including parotid gland resection, neck dissection, and manipulating the temporomandibular joint (TMJ).

\section{Results:}

\section{Vascular supply and drainage for auricle (cadaveric dissection) (Figure 2):}

We dissected cadavers to confirm the vascular structures related to the auricle (Fig. 2A). The main arterial supply to this area is the branches of the external carotid artery: posterior auricular artery and superficial temporal artery. The external carotid artery (ECA) gives off the posterior auricular artery just before it divides into the maxillary and superficial temporal arteries, which are terminal branches of the ECA in the parotid gland. These arteries play an important role in vascularizing the auricle. Separating the parotid and the auricle/external auditory canal exposes the middle auricular artery (Fig. 2B). The superior auricular artery diverges from the superficial temporal artery and courses superior to the auricle, forming the arterial network with the posterior auricular artery (Fig. 2B). Partial removal of the preauricular parotid gland exposes the course of the middle and inferior auricular artery and posterior auricular artery (Fig. 2C). The superior, middle, and inferior auricular arteries branch from the superficial temporal artery and supply the lateral surface of the auricle. The posterior auricular artery passes anterior to the mastoid process and supplies the mastoid branch to the skin on the mastoid process (Fig. 2D), which passes upward to supply the medial surface of the auricle with three main branches: superior, middle, and inferior (Fig. 2E). There are several perforating branches that penetrate the cartilage to form a complex arterial network between the lateral and medial surface of the auricle (Fig. 2F). The venous drainage from the auricle follows these arteries and connects to the superficial temporal vein anteriorly and the posterior auricular vein posteriorly. The venous plexus around the condyle of the mandible connects to the superficial temporal vein. The superficial temporal vein also receives venous flow from the middle temporal, parotid, and transverse facial veins and forms the retromandibular vein (Fig. 1C, 2G, and $\mathrm{H}$ ). The middle temporal vein courses beneath the superficial layer of the deep temporal fascia and joins the superficial temporal vein approximately $1 \mathrm{~cm}$ 
below the upper border of the zygomatic root. It connects the supraorbital vein and the superficial temporal vein. The retromandibular vein divides into two branches. The anterior branch passes forward and joins the facial vein to form the common facial vein, which ultimately drains into the internal jugular vein. The posterior auricular vein descends behind the auricle and joins the posterior branch of the retromandibular vein to form the external jugular vein (Fig. 2I).

\section{Auricular complication after en bloc temporal bone surgery}

The clinical characteristics of 64 patients are listed in Table 1. The patients included 22 men $(34.4 \%)$ and 42 women $(65.6 \%)$, with a median age of 67 years (range: 33-84 years). We included 57 SCC cases $(89.1 \%)$ and $7 \mathrm{ACC}$ cases (10.9\%). In 64 cases, 21 cases $(32.8 \%)$ had a history of radiotherapy at the locoregional site before surgery. Incision types (Fig. 3A) around the auricle were as follows: $\mathrm{S}$ shape (2 cases), C shape (17 cases), large shape (24 cases), inferior $\mathrm{C}$ shape (13 cases), upper $\mathrm{C}$ ( 7 cases), and preauricular incision (1 case). The incision around the EAC orifice varied (Fig. 3B). We used a small circumference incision around the orifice (Type a) for 58 cases. One case had the circumference incision crossing the crus helix (Type b). We applied the circumference incision, including the preauricular skin (Type c) to two cases. We sacrificed the inferior half of the auricular in three cases (Type d).

Three of 64 cases (4.7\%) developed postoperative auricular complications (Fig. 4) and all cases showed venous congestion (case 26, 27, and 43). We found auricular congestion and started mechanical bloodletting within 1 hour postoperatively in case 26 and 27. Several full thickness skin defect wounds (5 mm diameter) were created in the auricle, without exposing the cartilage, and covered with heparin-soaked gauze sequentially to maintain active bleeding from the wounds. Mechanical bloodletting enabled recovery from auricular congestion and the auricles in both cases were salvaged. However, for case 27 , a $1 \mathrm{~cm} \times 2 \mathrm{~cm}$ postauricular skin segment developed necrosis and required debridement. For case 43, just pin pricking was applied postoperatively to confirm the auricular congestion, and mechanical bloodletting was not applied. This resulted in auricular necrosis due to venous congestion. We performed debridement of necrotic tissue and finally the majority of the auricle was sacrificed.

To reveal the risk factors associated with auricular complications of temporal bone resection, we examined its relationship with the categorical variables, including neck dissection, pre-/infra-auricular lymph node dissection, parotidectomy, TMJ manipulation, and history of radiotherapy. We found that pre-/infra-auricular lymph node dissection was correlated with auricular complications, with statistical significance.(Table 2) The relationship between the type of microvascular anastomosis for reconstructing the auricular complication remains unclear due to an insufficient number of cases for analysis. The facial artery was used for vascular anastomosis for reconstruction in three cases, but these cases did not experience auricular complications.

\section{Discussion:}

Many groups have reported on the vascular flow of the auricle. (3-6) Blood supply depends on two branches of the external carotid arteries: 1) the superficial temporal artery; and 2) the posterior auricular artery. These branches form the dense arterial network perforating the cartilage. Recently, Zilinsky re-evaluated the arterial supply of the auricle. Superior and inferior auricular branches of the superficial temporal artery mainly supply the helical rim of the auricle.(6) The upper third of the helical rim was also supplied from non-performing branches of the posterior auricular artery. The perforators of the posterior auricular artery supply the concha, inferior crus, triangular fossa antihelix, and the ear lobe.(6) Venous drainage occurs through the accompanying veins of each artery.

LTBR and STBR are popular surgical methods for en bloc resection of temporal bone malignancy. It is clear that arterial supply to the auricle is significantly impaired when en bloc resection combined with parotidectomy is performed or when the superficial temporal artery is sacrificed. The frequency of auricular complication has not been elucidated. To our knowledge, Carpenter al. first reported ear complications in temporal bone surgery.(2) They followed 32 patients who underwent lateral skull base surgery, including parotid surgery, subtemporal fossa surgery, and temporal bone surgery. Postoperative necrosis was found in 3 of the 32 cases. However, that study reported no statistically significant risk of avascular necrosis when 
comparing preauricular and postauricular approaches, suggesting that no detailed study had previously identified a significant relationship with surgical procedures.

Simple LTBR can logically preserve the arterial supply of the auricle, and theoretically preserve anterior venous drainage of the superficial temporal vein. However, with STBR or LTBR, along with additional procedures, including parotidectomy and manipulation of the TMJ, it is impossible to preserve the main arterial branches to the auricle and both the main anterior and posterior venous drainage routes. We examined 34 cases who had undergone these procedures, and three of them showed postoperative vascular insufficiency in the auricle. However, sacrificing main arterial flow did not lead to ischemic auricular necrosis in any cases. Generally, the facial region has an abundant arterial network. The result of our study suggests the auricles had sufficient blood supply via the anastomosis connecting the facial arterial network. In our study, only one case showed postoperative auricular necrosis after auricular congestion, due to insufficient bloodletting after surgery. Appropriate postoperative bloodletting recovered auricular congestion and preserved the auricle cosmetically in the other two cases with postoperative vascular insufficiency in the auricle. These facts imply that venous drainage is crucial for preserving the auricle after temporal bone resection.

Veins in the face, which include the superior ophthalmic and facial veins, contain valves that determine blood flow direction. $(7,8)$ Zhang et al. mentioned that blood flow in the face is (1) caudal toward the internal jugular vein in the inferior part of the facial vein; (ii) normally toward the cavernous sinus in the superior ophthalmic vein; and (iii) into either the facial vein or the superior ophthalmic vein from the angular vein.(8) If the two main drainage routes from the auricle are sacrificed after temporal bone resection, the venous network in the face may be important for maintaining venous flow from the auricle. Hosokawa et al. recommend parotidectomy when the tumour invades the anterior-inferior wall of the canal.(9) If an early-stage tumour invaded this wall, we applied partial resection of the parotid gland and soft tissue abutting the canal. Furthermore, total parotidectomy is recommended for advanced T3 and T4 cases.(10, 11) The superficial temporal artery emerges from the superior border of the parotid gland and courses superolaterally between the TMJ and the tragus, and cross-superficially to the zygomatic process accompanied by the superficial temporal vein. Therefore, dissecting the infra-/pre-auricular region and manipulating the TMJ results in sacrificing the anterior drainage route from the auricular to the superficial temporal vein. To elevate the auricle, the posterior drainage route to the posterior auricular vein needs to be divided in all temporal bone resection cases. During neck dissection, common facial veins are often ligated. These cause a dramatic change in the venous flow related to the auricle. Unlike with arterial flow, it is likely that the valves in veins make it difficult for the venous drainage system to adapt to changes in blood flow after temporal bone resection. When pre-/infra-auricular lymphadenopathy is found preoperatively, the tissue around the lymph node needs to be sufficiently dissected to achieve adequate surgical margins. Therefore, temporal bone resection combined with total, superficial, or partial parotidectomy or with TMJ manipulation leads to sacrificing the main venous drainage from the auricle to the superficial temporal vein and the posterior auricular vein. In addition, careful dissection of the subcutaneous tissue around the lymphadenopathy potentially damages the venous network in the subcutaneous layer, which can worsen auricular congestion postoperatively.

Our analysis showed that cases with pre-/infra-auricular lymph node dissection had a statistically significant risk of auricular complication in the temporal bone resection (Fig. 5). Neck dissection, parotid gland resection TMJ manipulation and history of radiation therapy can worsen the venous drainage, theoretically. In addition, our study suggest that pre-/infra-auricular lymph node dissection had most impact to the worsens venous drainage leading to auricular congestion. A clear consensus on the guidelines for treating external ear congestion is lacking. Tissue milking, pin pricking,(12) use of medical leeches,(13, 14) mechanical leeching, (15) and chemical leeching(16) are the current treatment methods for venous congestion. In our two cases, mechanical bloodletting effectively salvaged the auricular necrosis. When the colour of the auricle changes during surgery, either 1) arterial spasm or 2) venous flow obstruction could be the cause. If ischemia due to artery spasm is observed, bleeding from the wound is confirmed after some time. If congestion is observed, it is important to check whether congestion can be recovered by pinprick, bloodletting and so on. Sufficient bloodletting should be initiated as soon as possible following confirmation of auricular congestion by the surgeon. 
Our study involved several limitations, including small sample size and its retrospective nature. In addition, our sample was comprised of only 63 patients. Further studies are needed to confirm the cause and risk factors associated with auricular complications.

\section{Conclusion:}

Temporal bone resection can cause auricular congestion due to insufficient venous outflow. An immediate and continuous application of bloodletting can replace insufficient venous drainage after temporal bone resection and prevent auricle necrosis.

\section{References}

1. Moody SA, Hirsch BE, Myers EN. Squamous cell carcinoma of the external auditory canal: an evaluation of a staging system. Am J Otol. 2000;21(4):582-8.

2. Carpenter PS, Burgette RC, Leonetti JP, Marzo SJ. Auricular complications in parotid, temporal bone, infratemporal fossa, and lateral skull base surgery. Ear, nose, \& throat journal. 2017;96(2):E27-E31.

3. Henoux M, Espitalier F, Hamel A, Dreno B, Michel G, Malard O. Vascular Supply of the Auricle: Anatomical Study and Applications to External Ear Reconstruction. Dermatol Surg. 2017;43(1):87-97.

4. Pinar YA, Ikiz ZA, Bilge O. Arterial anatomy of the auricle: its importance for reconstructive surgery. Surgical and radiologic anatomy : SRA. 2003;25(3-4):175-9.

5. Wilson C, Iwanaga J, Simonds E, Yilmaz E, Oskouian RJ, Tubbs RS. The Conchal Vascular Foramen of the Posterior Auricular Artery: Application to Conchal Cartilage Grafting. Kurume Med J. 2018;65(1):7-10.

6. Zilinsky I, Erdmann D, Weissman O, Hammer N, Sora MC, Schenck TL, et al. Reevaluation of the arterial blood supply of the auricle. J Anat. 2017;230(2):315-24.

7. Nishihara J, Takeuchi Y, Miki T, Itoh M, Nagahata S. Anatomical study on valves of human facial veins. Journal of cranio-maxillo-facial surgery : official publication of the European Association for Cranio-MaxilloFacial Surgery. 1995;23(3):182-6.

8. Zhang J, Stringer MD. Ophthalmic and facial veins are not valveless. Clin Exp Ophthalmol. 2010;38(5):50210.

9. Hosokawa S, Mizuta K, Takahashi G, Okamura J, Takizawa Y, Hosokawa K, et al. Surgical approach for treatment of carcinoma of the anterior wall of the external auditory canal. Otol Neurotol. 2012;33(3):450-4.

10. Lee JM, Joo JW, Kim SH, Choi JY, Moon IS. Evidence Based Tailored Parotidectomy in Treating External Auditory Canal Carcinoma. Sci Rep. 2018;8(1):12112.

11. Xie B, Wang M, Zhang S, Liu Y. Parotidectomy in the management of squamous cell carcinoma of the external auditory canal. European archives of oto-rhino-laryngology : official journal of the European Federation of Oto-Rhino-Laryngological Societies (EUFOS) : affiliated with the German Society for OtoRhino-Laryngology - Head and Neck Surgery. 2020.

12. Pafitanis G, Chen HC. The Pinprick Test: Key Considerations in Execution of Skin Flap Perfusion Testing. Plast Reconstr Surg Glob Open. 2019;7(9):e2370.

13. Facchin F, Lancerotto L, Arnez ZM, Bassetto F, Vindigni V. Leeching as Salvage Venous Drainage in Ear Reconstruction: Clinical Case and Review of Literature. Plast Reconstr Surg Glob Open. 2018;6(11):e1820.

14. Sig AK, Guney M, Uskudar Guclu A, Ozmen E. Medicinal leech therapy-an overall perspective. Integr Med Res. 2017;6(4):337-43.

15. Nath RK, Kraemer BA, Azizzadeh A. Complete ear replantation without venous anastomosis. Microsurgery. 1998;18(4):282-5. 
16. Barnett GR, Taylor GI, Mutimer KL. The "chemical leech": intra-replant subcutaneous heparin as an alternative to venous anastomosis. Report of three cases. British journal of plastic surgery. 1989;42(5):556-8.

\section{Figure legends}

Figure 1. Flow chart of patient selection.

Figure 2. Vascular supply of the left auricle.

(A) Structures around the auricle exposed following skin removal. (B) Exposed structures related to the auricle in the preauricular region. (C) Removal of the preauricular parotid exposing the course of vascular structures in the preauricular region. (D) Enlarged view of the infra-auricular region in C. (E) Exposed course of the posterior auricular artery. (F) Exposed perforating branches of the posterior auricular artery. $(\mathrm{G}-\mathrm{H})$ The auricle, parotid gland, and condyle of the mandible are shown to reveal the venous network medial to the parotid gland $(\mathrm{G})$. Removal of the mandibular condyle shows the relationship between the superficial temporal vein and the parotid gland $(\mathrm{H})$. The venous plexus around the condyle connects to the superficial temporal vein forming the retromandibular vein. (I) The course of the retromandibular vein and its related venous connection. The part of the retromandibular vein between the asterisks is removed to show the deeper structures.

A., artery; Auric., auricular; Br., branch; Com., common; Comm., communication; EAM, external auditory meatus; EJV, external jugular vein; Gl., gland; Gr., Great; IJV, internal jugular vein; Inf., inferior; Lat., lateral; Lt., left; M., muscle; Max., maxillary. Mid., middle; N., nerve; Occip., occipital; Post., posterior; Ptery., pterygoid; Retromand., retromandibular; Rt. right; STA, superficial temporal artery; SCM, sternocleidomastoid; STV, superficial temporal vein; Sup., superior; TMJ, temporomandibular joint; Temporopar., temporoparietal

Figure 3. Variation of skin incisions for temporal bone surgery

(A) Various types of pre- and post-auricular skin incisions. (B) Variations of skin incision methods around the orifice of the external auditory meatus.

Figure 4. Representative cases of auricular complication following temporal bone resection. Pin pricking was insufficient for bloodletting, resulting in auricular necrosis due to the congestion (upper row). Insert (upper right corner): Case 43 at POD9, showing the appearance of the auricle following necrotomy. Mechanical stabs covered with heparin-soaked gauze improve auricular congestion and prevent auricular necrosis (middle and lower rows).

Figure 5. Dissection of the pre- or infra-auricular lymph node dissection are one of the risk factors inducing auricular complications following temporal bone surgery.

\section{Hosted file}

Table 1.pdf available at https://authorea.com/users/382721/articles/498620-auricularcomplications-following-temporal-bone-resection-for-temporal-bone-malignancies-aclinical-consideration

\section{Hosted file}

Table 2.pdf available at https://authorea.com/users/382721/articles/498620-auricularcomplications-following-temporal-bone-resection-for-temporal-bone-malignancies-aclinical-consideration 


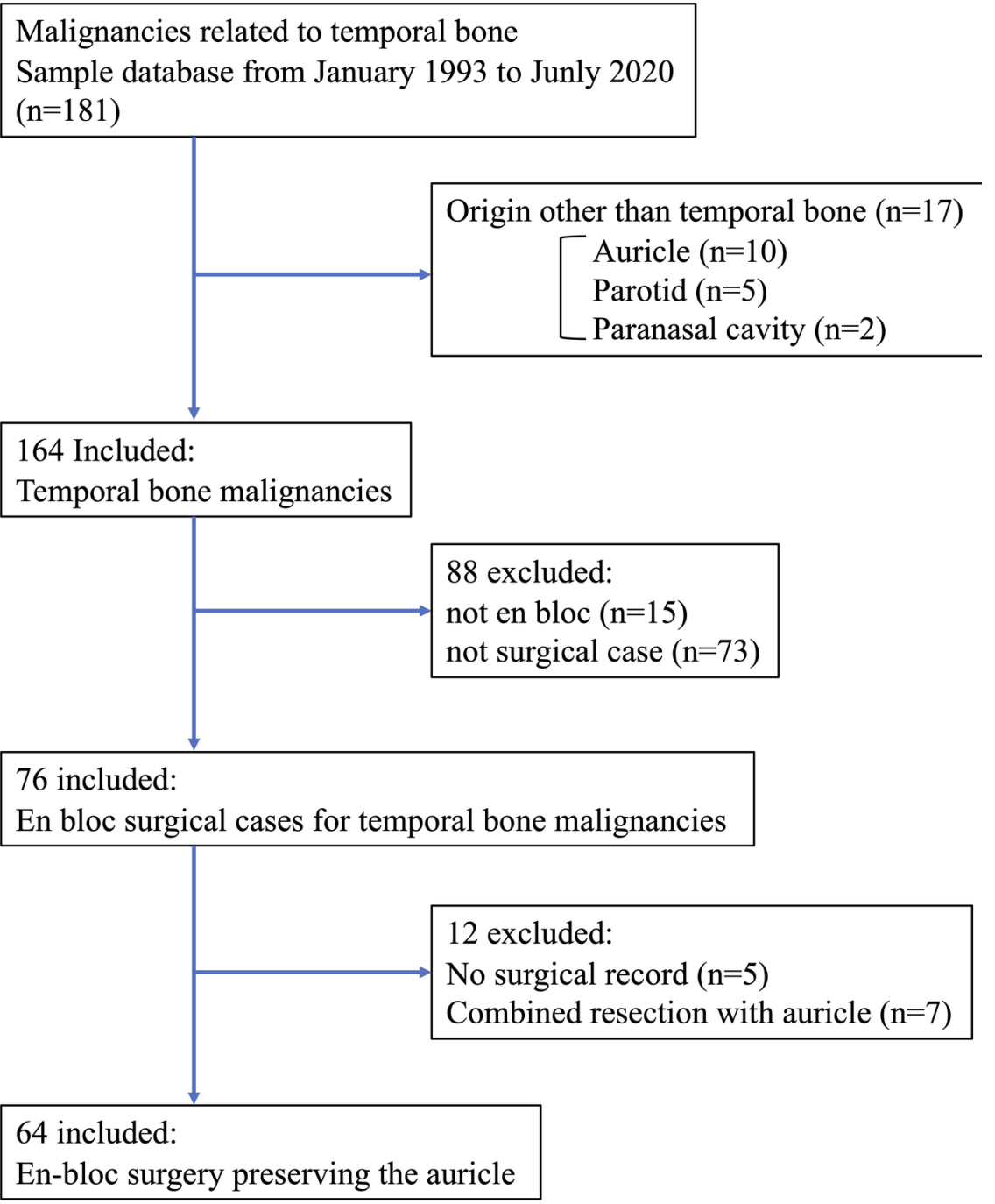



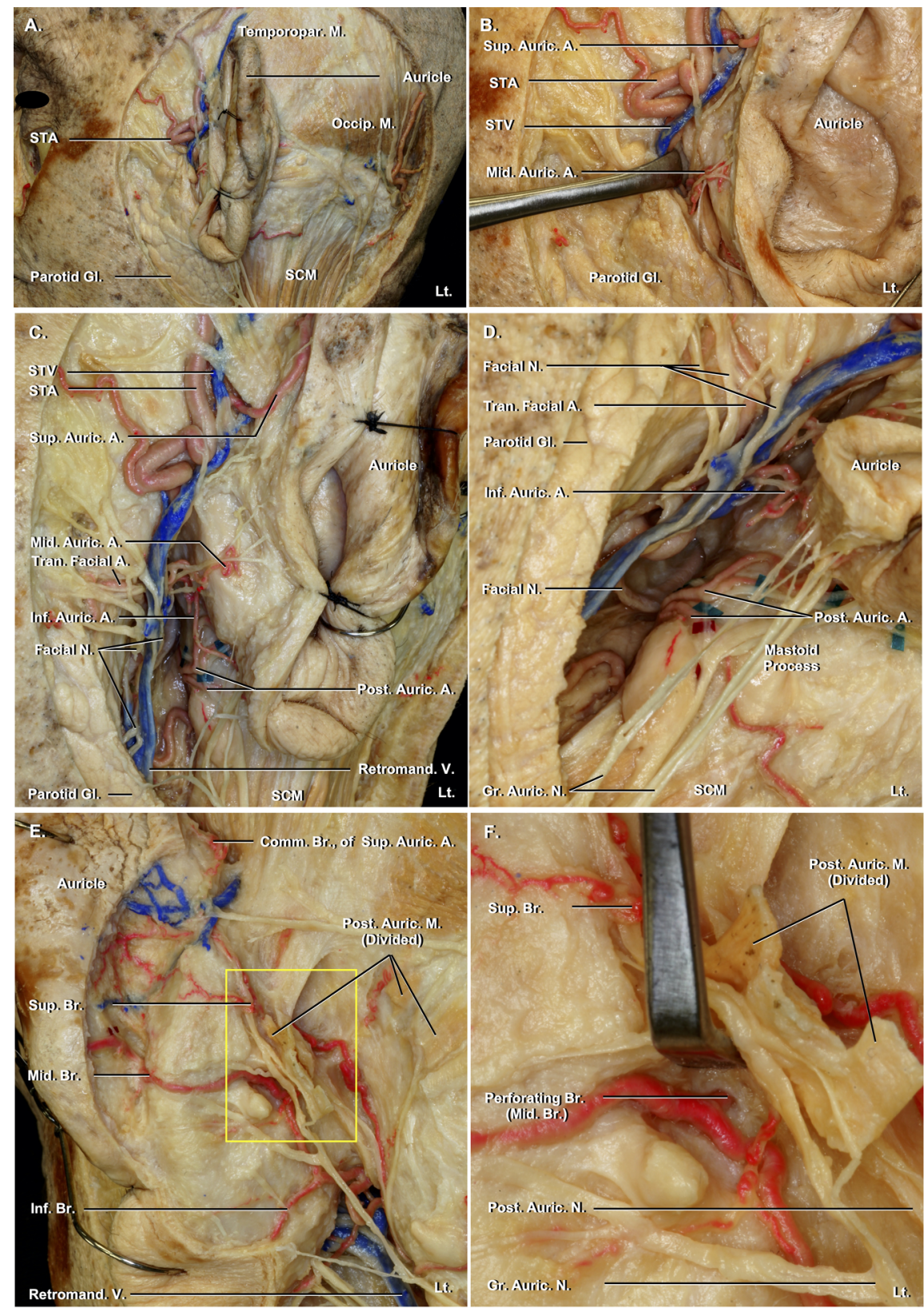

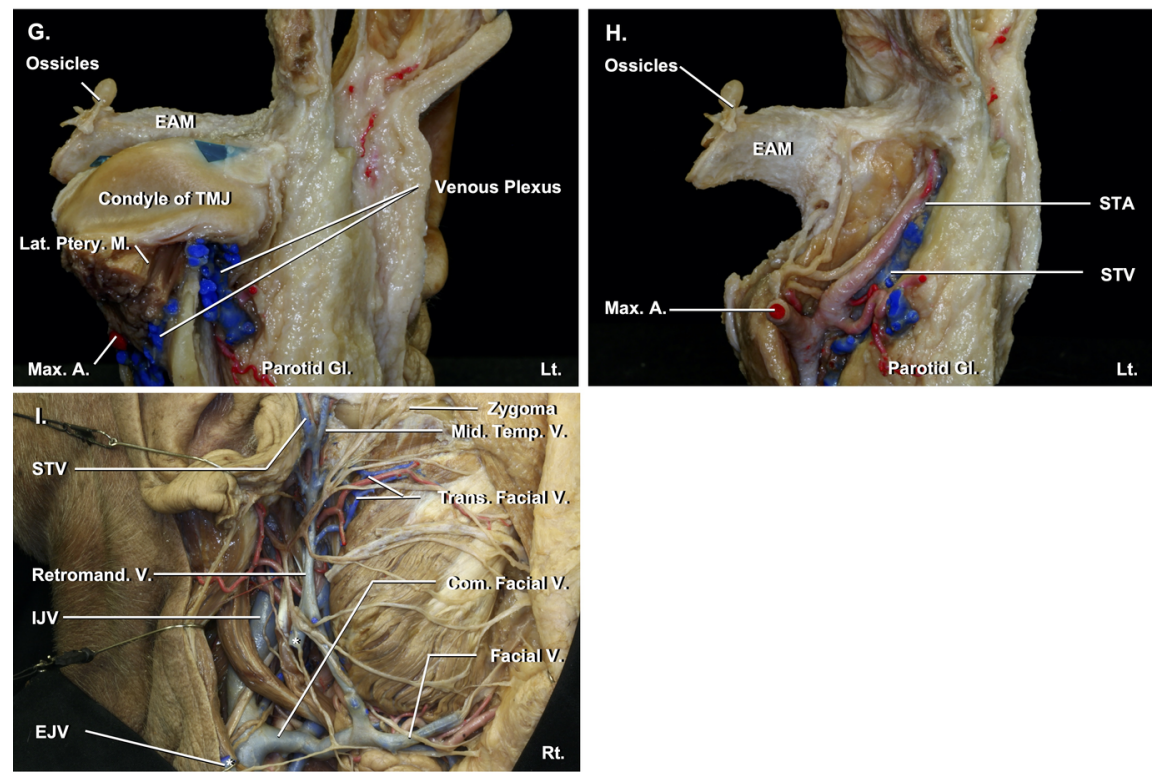

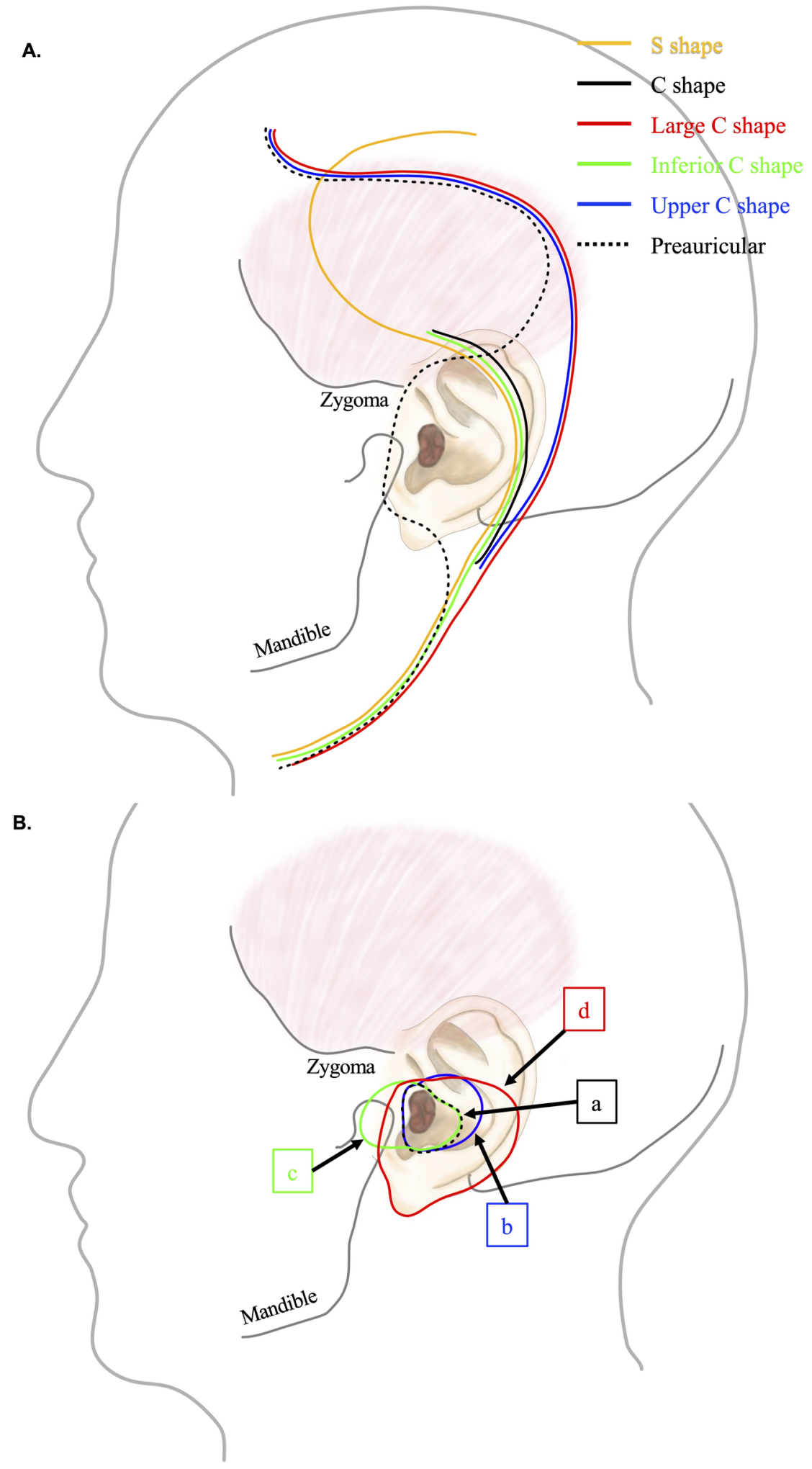

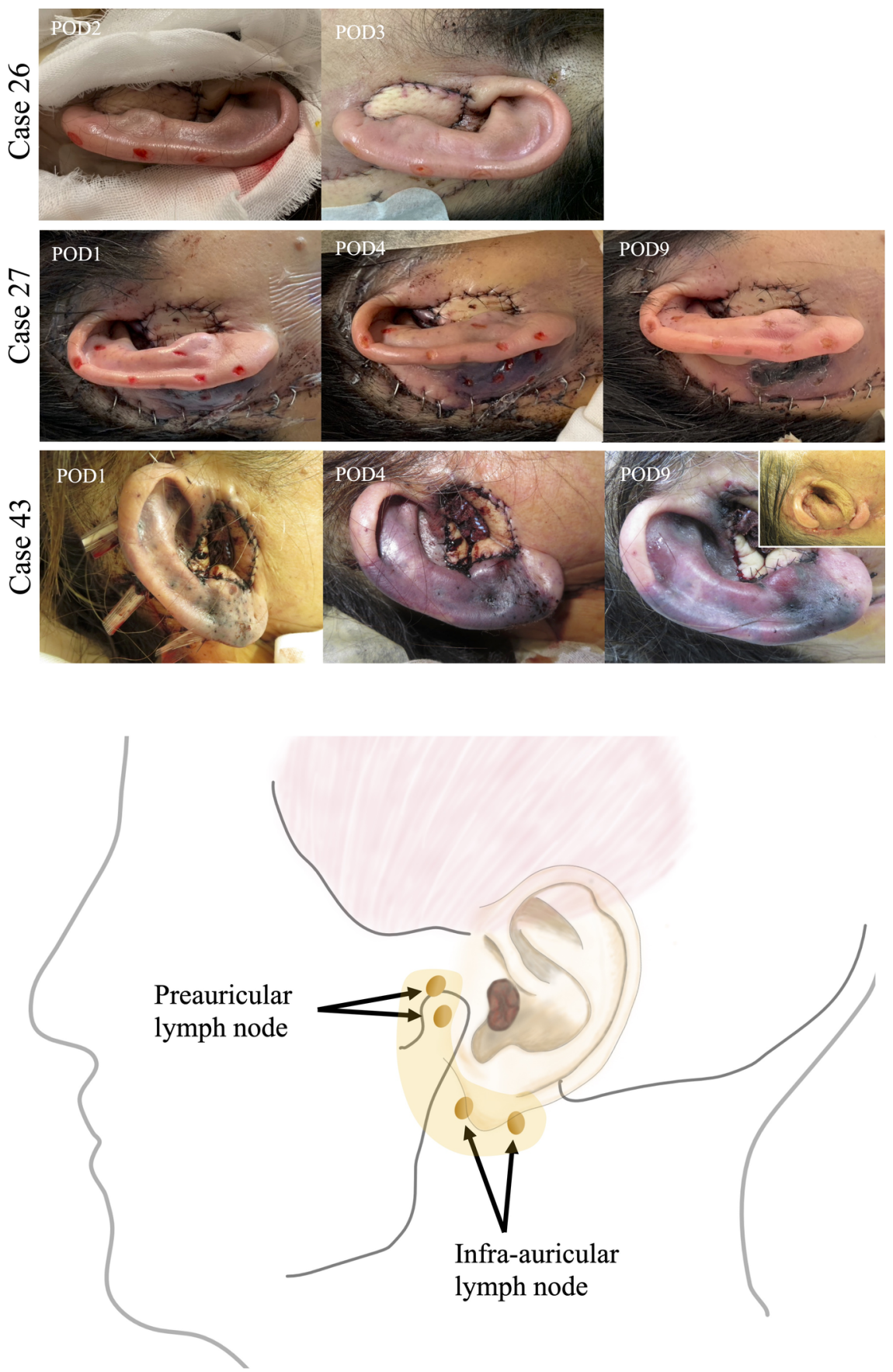